\title{
Sex differences in navigation strategy and efficiency
}

\author{
Alexander P. Boone ${ }^{1} \cdot$ Xinyi Gong ${ }^{1} \cdot$ Mary Hegarty ${ }^{1}$ \\ Published online: 22 May 2018 \\ (C) Psychonomic Society, Inc. 2018
}

\begin{abstract}
Research on human navigation has indicated that males and females differ in self-reported navigation strategy as well as objective measures of navigation efficiency. In two experiments, we investigated sex differences in navigation strategy and efficiency using an objective measure of strategy, the dual-solution paradigm (DSP; Marchette, Bakker, \& Shelton, 2011). Although navigation by shortcuts and learned routes were the primary strategies used in both experiments, as in previous research on the DSP, individuals also utilized route reversals and sometimes found the goal location as a result of wandering. Importantly, sex differences were found in measures of both route selection and navigation efficiency. In particular, males were more likely to take shortcuts and reached their goal location faster than females, while females were more likely to follow learned routes and wander. Self-report measures of strategy were only weakly correlated with objective measures of strategy, casting doubt on their usefulness. This research indicates that the sex difference in navigation efficiency is large, and only partially related to an individual's navigation strategy as measured by the dual-solution paradigm.
\end{abstract}

Keywords Navigation $\cdot$ Strategy $\cdot$ Efficiency $\cdot$ Sex differences

Imagine a meeting is about to take place at a specific location. The meeting participants show up and the meeting starts; a few people trickle in. While everyone got to the meeting (nearly) on time, the meeting participants likely did not plan their route to the meeting in the same manner, nor did they take the same route. For instance, one person may have taken a shortcut through a building, while another took a habitual, less direct route. These individual differences are an important aspect of understanding how humans navigate. An intriguing part of this puzzle is that differences are found in how men and women report thinking about space and in performing navigation tasks. Here, we investigate sex differences in navigation strategy, while also exploring how objective assessment of navigation strategy relates to self-reports of strategy and measures of environmental spatial ability.

Alexander P. Boone

Boone@psych.ucsb.edu

1 Department of Psychological and Brain Sciences, University of California, Santa Barbara, Santa Barbara, CA 93106, USA
Research in human spatial cognition has revealed large individual differences in ability to perform spatial tasks in both real and virtual environments ${ }^{1}$ (Chrastil \& Warren, 2015; Hegarty, Montello, Richardson, Ishikawa, \& Lovelace, 2006; Labate, Pazzaglia, \& Hegarty, 2014; Lawton, Charleston, \& Zieles, 1996; Weisberg, Schinazi, Newcombe, Shipley, \& Epstein, 2014). For instance, some individuals are better at pointing to unseen locations, drawing sketch maps of, or taking efficient routes in well-known or recently learned environments. These individual differences often depend on the type of knowledge that a person has constructed from their navigation experience. Spatial cognition researchers make distinctions between landmark, route, and survey knowledge. Landmark knowledge, the most basic

\footnotetext{
${ }^{1}$ Virtual environments have the advantage of enabling researchers to control navigation experience without extraneous variables that are difficult to control in real environments (e.g., position of the sun, weather conditions, construction). Navigation in desktop environments differs from real-world navigation in that it is not accompanied by proprioceptive cues that one receives while navigating in a real environment (Hegarty et al., 2006). Despite the absence of these cues, researchers have found relatively high correlations between navigating in real and virtual environments (Richardson, Montello, \& Hegarty, 1999) and research in virtual environments has replicated basic findings of real environment navigation (e.g., Weisberg et al., 2014).
} 
level of environmental knowledge, enables one to recognize key landmarks. Landmark knowledge alone is not useful for navigation. For instance, one might recognize the Saint Louis Gateway Arch by sight, but this alone does not allow you to know how to get to Busch Stadium. Route knowledge is information regarding the sequences of paths and turns needed to travel between locations. With route knowledge, one might know to walk towards the Old Courthouse and turn left until Busch Stadium is in view. Finally, survey knowledge is knowledge of the layout of the environment, including metric distances and directions between landmarks. People with survey knowledge of an environment can navigate to a location in that environment, using novel, unlearned routes (i.e., shortcuts) and point accurately to locations that are currently out of view.

While landmark, route, and survey knowledge were initially proposed to develop sequentially (Siegel \& White, 1975), recent evidence has suggested that all three forms of knowledge are developed gradually, but there are large individual differences in rate of acquisition. Ishikawa and Montello (2006) taught participants two routes in a suburban neighborhood before indicating a connecting path between the routes. Participants were asked to point to landmarks on each route and across routes. They showed large individual differences in people's ability to learn the environmental layouts such that the best navigators showed good survey knowledge (as measured by pointing to unseen locations and sketch mapping) after the first learning experience, and the worst navigators were still at chance level on these tasks after 10 exposures. These results were recently replicated in a virtual environment (Weisberg et al., 2014). Furthermore, in an fMRI study using a similar paradigm, Schinazi, Nardi, Newcombe, Shipley, and Epstein (2013) showed that individual differences in largescale environment layout learning and integration are in part driven by differences in hippocampal volume. Specifically, those with a larger right posterior hippocampus were more flexible in spatial knowledge expression, such as pointing to unseen locations. Expert navigators, such as taxi drivers in London, also show larger hippocampi relative to control participants (Maguire, Woollett, \& Spiers, 2006; Woollett \& Maguire, 2011). Taken together, these findings indicate that there are large individual differences in the ability to construct survey knowledge of an environment from the experience of taking routes in the environment and this is related to hippocampal volume.

In addition to these general individual differences, there are differences between men and women when performing largescale spatial tasks in real environments. A male advantage is often found in tasks that require survey knowledge (Coluccia \& Louse, 2004). For example, males perform better in pointing to unseen locations in a known environment (Ishikawa \& Montello, 2006), in remaining oriented to compass directions in unfamiliar environments (Sholl, Acacio,
Makar, \& Leon, 2000), in keeping track of the starting point while taking a circuitous walk (Silverman et al., 2000), and following navigation instructions that use cardinal directions and metric distances (Saucier et al., 2002). Some of the most pronounced sex differences are found in measures of navigation efficiency (e.g., time to navigate to a goal location). For example, males are significantly faster to escape a virtual maze (Grön, Wunderlich, Spitzer, Tomczak, \& Riepe, 2000; Moffat, Hampson, \& Hatzipantelis, 1998) and make fewer moves navigating through a wire-frame virtual environment (Cutmore, Hine, Maberly, Langford, \& Hawgood, 2000). In contrast, there are no systematic sex differences in tasks that can be accomplished with route and landmark knowledge, such as when learning from a map, retracing a learned route, or remembering landmarks along a route (Coluccia \& Louse, 2004; Montello, Lovelace, Golledge, \& Self, 1999; Saucier et al., 2002).

Sex differences are also found when people report their daily navigation strategies. Males more often report using survey-like strategies, such as taking shortcuts and focusing on distal landmarks to navigate, whereas females more often report using route-based strategies of following well-learned routes and depending on local landmarks (Coluccia \& Louse, 2004; Lawton, 1994, 1996; Lawton \& Kallai, 2002). Selfreport measures have the limitation that they rely on people having accurate metacognitive knowledge of their own abilities and strategies, so it is important to validate self-reports against objective measures of navigation performance. However, there has been relatively little research that has used objective measures of navigation strategies or that has related self-reported strategies to objective measures of the strategies that people adopt when they actually navigate.

Although there has been much research on the topic of strategy differences between men and women in navigation tasks, many studies on this topic (e.g., Sholl et al., 2000; Silverman et al., 2000) have examined the relative abilities of the sexes to perform route-based and survey-based strategies, rather than studying what strategies people actually use. The clearest evidence to date that men and women differ in strategy use comes from studies of a desktop virtual environment version of the Morris water-maze task, a task often used in the animal literature, which involves learning the location of hidden platform under water. Astur and colleagues (Astur, Ortiz, \& Sutherland, 1998; Astur, Tropp, Sava, Constable, \& Markus, 2004) found that men and women differed in their strategy on this task such that after a learning phase, men navigated directly to the hidden platform whereas women used a variety of strategies, such as searching in a circular or zig-zag pattern. A later study indicated that, when only given geometrical cues to find a learned but unseen platform location in a virtual Morris water maze, males significantly outperform females in finding the platform; however, when given stable landmark cues to navigate by, males and females show 
similar performance (Sandstrom, Kaufman, \& Huettel, 1998), suggesting that women depended more on landmarks (see also Lambrey \& Berthoz, 2007).

While studies using the virtual Morris water-maze task have shown that women depend more on landmarks while men depend more on room geometry, we cannot generalize from strategy differences on this task to all navigation tasks. First, Astur et al. (2004) found that performance on a virtual Morris water-maze task was not correlated with performance on a virtual radial-arm maze task, although these tasks are often assumed to be comparable measures of navigation. Second, dependence on landmarks versus more geometric cues is highly task dependent. For example, Foo, Warren, Duchon, and Tarr (2005) found that on a triangle-completion task, both men and women depended on landmarks when they were available. Moreover, Cutmore et al. (2000) varied whether cardinal directions or landmarks were available to men and women in a virtual navigation task and found that men were more likely to use the landmarks while neither men nor women were affected by the cardinal direction cues. Therefore, it is important to examine objective evidence for strategy differences between men and women in a range of navigation tasks.

Recently there has been new interest in the development of objective measures of individual differences in navigation strategy (Furman, Clements-Stephens, Marchette, \& Shelton, 2014; Iaria, Petrides, Dagher, Pike, \& Bohbot, 2003; Marchette et al., 2011). For instance, in the dual-solution paradigm (DSP; Marchette et al., 2011), participants first learn a route through a desktop virtual environment and then must navigate between previously learned locations. Participants typically take either a learned route or a novel shortcut on each trial to arrive at the goal location. Using this task, strategy is measured via the solution index (SI), which is equal to the number of trials in which a shortcut is taken divided by the number of successful trials in which either a shortcut or the learned route is taken. This task has highlighted marked individual differences in navigation strategy, such that some individuals primarily use shortcuts, some primarily use learned routes, and many participants fall between these extremes (Furman et al., 2014; Marchette et al., 2011; Weisberg \& Newcombe, 2016). Success in navigation to the goal is not related to navigation strategy. Further, fMRI analyses indicate that brain activations during DSP encoding trials predict later route selection such that participants showing greater hippocampal activations take shortcuts more often (Marchette et al., 2011). These results are consistent with the wider literature indicating that the hippocampus supports cognitive map or survey-based navigation (O'Keefe and Nadel, 1978; Packard \& McGaugh, 1996; Schinazi et al., 2013). Using shortcuts in this task is also related to spatial perspective-taking ability (Marchette et al., 2011). In a recent study with 28 participants, self-reported navigation strategy was found to moderately correlate with strategy (Furman et al., 2014).

\section{Introduction}

\section{The present study}

In two experiments, we utilize the DSP (Marchette et al., 2011) to examine whether there are sex differences in objectively assessed navigation strategy. Given the male advantage in tasks that depend on survey knowledge (Coluccia \& Louse, 2004) and sex differences in subjective measures of strategy (Lawton, 1996; Pazzaglia, Cornoldi, \& De Beni, 2000), we predicted more use of shortcut strategies by men and more use of route-based strategies by women. Based on previous research (Astur et al., 1998; Astur et al., 2004; Moffat et al., 1998; Grön et al., 2000), we also predicted that men would navigate more efficiently. Measuring both strategy and efficiency also enables us to examine how much efficiency differences are due to use of alternative strategies.

A secondary goal was to examine the relationship between strategy used in the DSP, a self-report measure of strategy, and measures of large-scale spatial ability, including self-reported sense of direction and perspective-taking ability. While selfreport measures of strategy correlate with measures such as pointing accuracy (Lawton et al., 1996), and one study revealed a relationship between the DSP and a subjective measure of strategy (Furman et al., 2014), the relationships between objective and subjective measures of strategy have not been studied in the context of sex differences.

\section{Experiment 1}

Experiment 1 examined sex differences in the DSP. We predicted that males would take more shortcuts and take less time to navigate to goal locations while females would take more learned routes and take more time to navigate to goal locations. Another goal was to explore the relationship between strategy and efficiency to evaluate the degree to which sex differences in efficiency could be accounted for by differences in strategy. The final goal was to evaluate the relation of these measures to standard measures, both objective and subjective, of large-scale spatial ability and strategy. We predicted that subjective and objective measures would be related and that taking shortcuts in the DSP would be related to measures of large-scale spatial ability.

\section{Method}

\section{Participants}

Participants were 72 University of California, Santa Barbara, undergraduates who participated in return for course credit. Four participants were excluded from analysis due to motion sickness $(N=2)$, colorblindness $(N=1)$, and experimenter 
error $(N=1)$. Sixty-eight participants (40 female, 28 male) were included in the final analyses.

\section{Materials}

The experiment was administered using a Dell Optiplex 9020 with an AMD Radeon 8570 graphics card computer running Windows 7 64-bit and presented through Vizard 4.0 (Santa Barbara, CA). The environment was displayed on a 24 -inch LCD monitor $(289.9 \times 531.4 \mathrm{~mm}$ display area $)$ with a refresh rate of $60 \mathrm{~Hz}$ at a resolution of $1920 \times 1080$. The viewing distance was approximately $1,000 \mathrm{~mm}$. The field of view in the virtual environment was set to 60 degrees.

Maze environment We created a VR training maze (see Fig. 1a) and a DSP learning maze, which were the same size and shape $(55 \times 55$ meters $)$ and had no dead ends. There were 12 nodes within the DSP learning maze, and landmark objects were placed in 11 of these nodes (see Fig. 1b). These objects were: chair, duck, telescope, large plant, soccer ball, stove/range, piano, car, wheelbarrow, harp, and wooden well. Each object was easily detectable against the concrete wall and could be seen in a single view. As Fig. 2 illustrates, basic concrete texture was used for the walls of the maze, and the sky was displayed as a flat light blue without clouds or a central light source to eliminate possible use of directional cues.

Psychometric measures of spatial ability The Money Road Map test (MRM; Money, Alexander, \& Walker, 1965) presented participants with a bird's-eye view of a street network map with a single dotted line marking a navigator's path. Participants were told to imagine travelling this path and mark " $\mathrm{R}$ " or " $\mathrm{L}$ " to indicate a left or right turn at each corner. Participants were given $30 \mathrm{~s}$ to mark as many turns as possible (out of 32), in order of the path,

a)

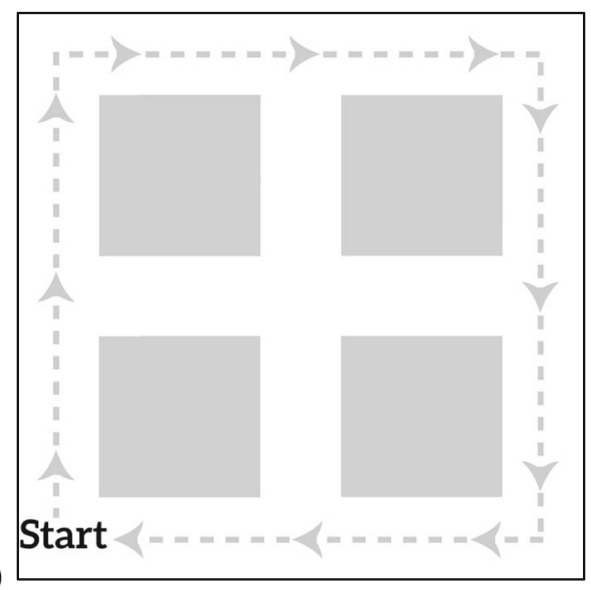

Fig. 1 VR training maze (a) and dual solution paradigm maze (b) structure modeled after Marchette et al. (2011). The starting location is marked with text, while the arrows indicates the direction of travel. Black without turning the sheet. The Spatial Orientation Test (SOT; Hegarty \& Waller, 2004) presented participants with an array of objects above an arrow circle. Text above the arrow circle instructed participants to imagine standing at one object while facing a second and then pointing to a third object in the array (e.g., "Imagine you are standing at the cat, facing the tree, point to the flower"). Participants were required to draw a line from the center of the arrow circle to the edge of the circle to indicate the designated relationship. The SOT consisted of 12 trials with a maximum time limit of 5 minutes. The measure of performance was the angular error averaged across trials. Incomplete trials were assigned an angular error of 90 degrees (chance performance).

Self-report measures The Santa Barbara Sense of Direction Scale is a self-report measure of environmental spatial abilities (Hegarty, Richardson, Montello, Lovelace, \& Subbiah, 2002). Participants are provided 15 statements such as "I very easily get lost in a new city" and rate their agreement with each on a scale of 1 to 7 . in which 1 is strongly agree and 7 is strongly disagree. The Lawton Wayfinding Strategy Scale (WSS; Lawton, 1994) asks participants to consider a recent trip they have taken to answer questions about how they approached navigation. The first two subscales separately assess orientation and route strategies, while the third scale assesses spatial anxiety. A 1 to 5 scale is provided, where 1 is extremely not typical of me and 5 is extremely typical of me. Finally, participants were asked to report on their video-game experience (none, little or some, moderate, a lot).

\section{Procedure}

After giving informed consent, participants were given the opportunity to practice with the active navigation controls (keyboard and mouse) within the VR training maze (see

b)

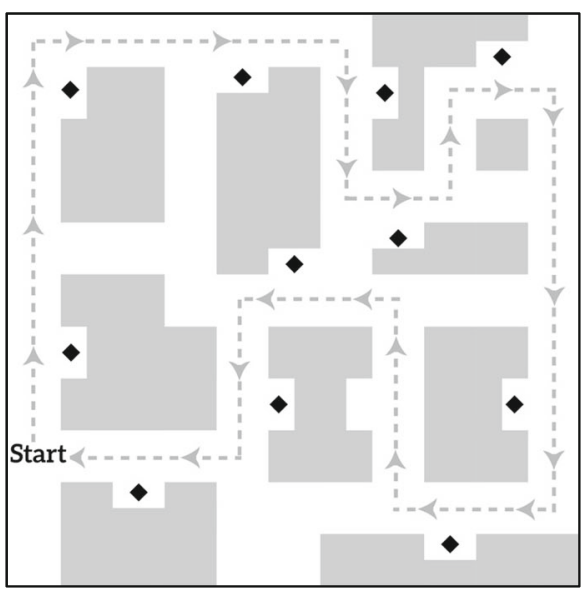

diamonds represent local landmark locations. Participants never saw the overhead representation 
a)

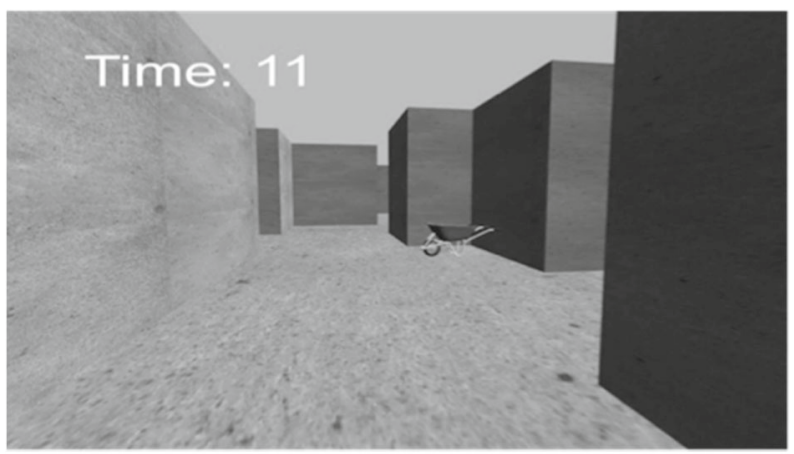

b)

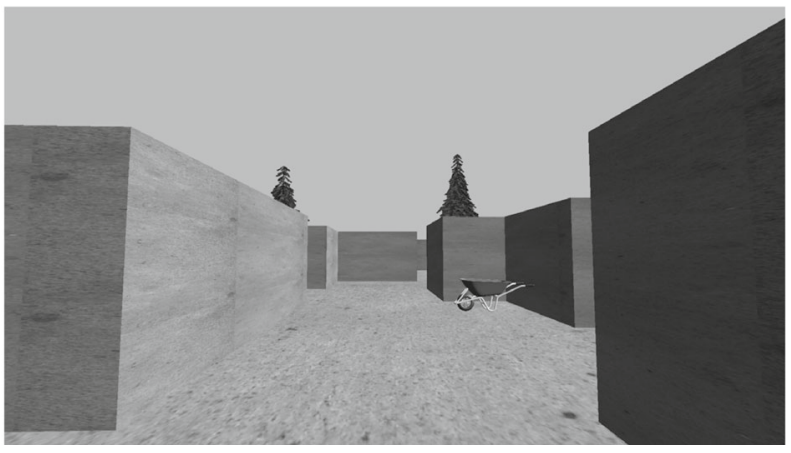

Fig. 2 Examples of views of the environment that a participant saw in the (a) timer condition of Experiment 1 and (b) in the distal landmark condition of Experiment 2

Fig. 1a) until they indicated they were comfortable with the controls. In the learning phase of the DSP (Fig. 1b), participants were asked to follow red arrows on the ground to navigate the maze, while taking note of the objects that they passed. An invisible wall blocked each corridor that was not on the learned path, but the view of the corridor was not obscured. Participants followed the route five times. Following a 30 -second break, participants were given instructions for the testing phase. Participants were placed in different locations along the learned route and were asked to navigate to another location within the maze. For example, in one trial, the person was placed near the wooden well and had to navigate to the stove (see Fig. 3). There were 24 total trials. Twelve trials were "shortcut available" trials, in which there was a shortcut (i.e., a path to the goal that was shorter than the learned route), six were "shortcut equivalent" trials, in which the shortest novel path and the learned route were equivalent in length. The final six trials served as catch trials, in which the learned route was actually the shortest path to the goal location.

For half of the participants, a countdown timer of 60 seconds was presented in the top right of the screen (see Fig. 2a). A trial ended when the participant reached the goal location or when $60 \mathrm{~s}$ elapsed, whichever was sooner. The other half of the participants did not see a timer, nor did the trial end at 60 seconds, but we only analyzed their performance within the first $60 \mathrm{s.}^{2}$

Next, participants were administered the Money Road Map Test (MRM), the Spatial Orientation Task (SOT; Hegarty \& Waller, 2004), the Santa Barbara Sense of Direction Scale (SBSOD; Hegarty et al., 2002), the Lawton Wayfinding Strategy Scale (WSS), including the spatial anxiety component (Lawton, 1994), and asked to rate their video-game experience. After completion of all tasks, participants were debriefed and dismissed.

\section{Coding}

A preliminary coding of the DSP navigation retrieval trials by two raters (blind to gender of the participants) revealed four strategies: taking a shortcut, taking the learned route, reversing the learned route, and wandering (repeating sections of the route until the goal was eventually found). Small deviations from the shortest path, learned routes, and reverse learned routes were allowed in a liberal coding scheme. For example, if a participant primarily followed the learned path but near the target landmark had a minor deviation from this path, that trial was coded as a liberal learned route. Figure $3 b$ and $d$ present examples of these categories. Interrater reliability was high $(k$ $=.96, p<.001$ ).

This initial coding scheme was then used to formalize the liberal coding scheme mathematically, with the general criteria that a shortcut was shorter than the learned route and had little overlap with the learned route. A shortcut was defined as a path that is no more than $84 \%$ the length of the learned route and in which less than $70 \%$ of the path taken was on the learned route. The mean length of trials coded as shortcuts was $64 \%$ of the learned route, and the average overlap of the shortcuts with learned route was 39\%. Trials were coded as following the learned route if participants traversed $70 \%$ or more of the learned route; on average, trials classified as learned route overlapped this route by $80 \%$. Similarly, trials coded as reversing the learned route overlapped this route by at least $70 \%$ and by $87 \%$ on average. Trials were classified as "wandering" when a major section of the participant's route was repeated, so that the route taken was longer than the learned route. Finally, trials that did not meet any of these criteria were classified as uncodable.

As in previous research, we computed a single, solution index measure (SI; Furman et al., 2014; Marchette et al.,

\footnotetext{
${ }^{2}$ In Experiment 1 , half of the participants were given a time limit of 60 seconds per trial, and a timer was displayed on the screen during their trials. There were 20 females and 14 males in each condition (timer vs. control). Participants in the timer condition did not differ from controls in any dependent measure. This is probably because the time limit of 60 seconds per trial turned out to be liberal, as the average time per trial was $19.38 \mathrm{~s}(S D=10.18)$. We considered only the first 60 seconds of each trial, regardless of whether the participant was in the timer or control condition and collapsed over timer conditions in all analyses presented here.
} 
a)

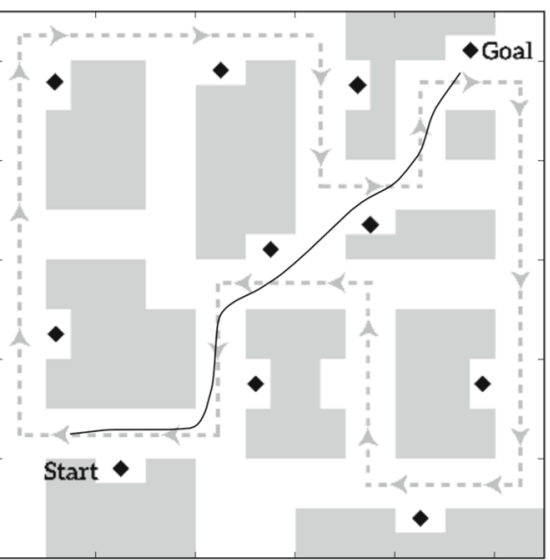

c)

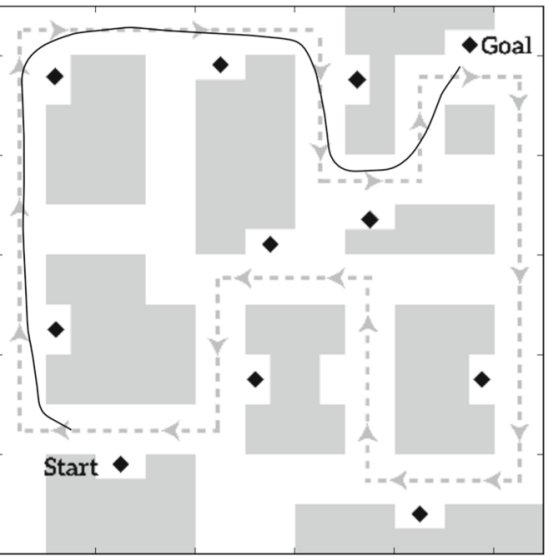

e)

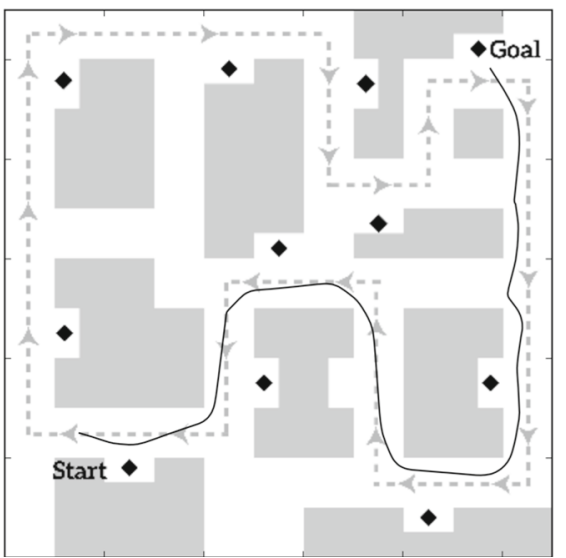

b)
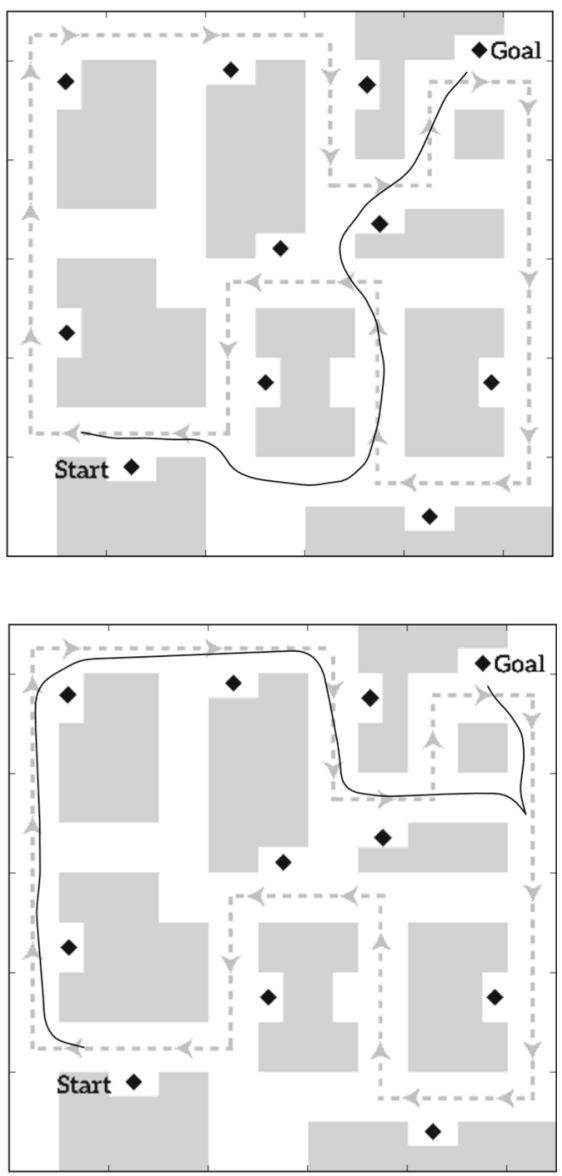

d)

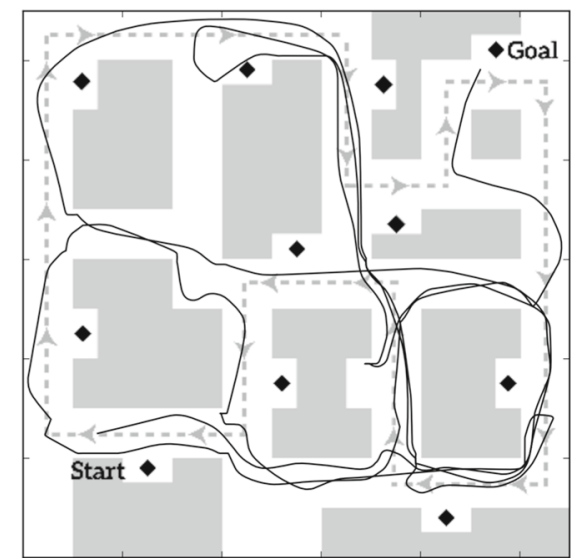

Fig. 3 Representative trial of each major category code. Note. a shortcut, $\mathbf{b}$ shortcut liberal, $\mathbf{c}$ learned, $\mathbf{d}$ learned liberal, $\mathbf{e}$ reversed learned, $\mathbf{f}$ wandering. Dotted line indicates the initially learned path. Black diamonds indicate the locations of landmarks in the environment

2011), which takes account of the number of shortcut trials divided by the sum of shortcuts and learned routes (including liberally coded trials) and produces a number on a scale of 0 (indicating all learned routes) to 1 (indicating all shortcuts). Reversals of the learned route and wandering were not considered in computing this index.

To assess navigation efficiency, time and distance travelled to the goal location on trial were computed for each trial, then
Z-score transformed and averaged across all shortcut available trials for each participant. This measure weights all trials equally and expresses navigation efficiency relative to other participants. There was a strong correlation between the distance and time efficiency measures, $r(66)=.98, p<.001$; therefore, we adopted time to find the goal as our measure of efficiency. Results do not differ if we use distance taken to the goal location. 


\section{Results}

Sex differences in ability and self-report measures Table 1 presents descriptive statistics for the psychometric and selfreport measures. As expected based on previous research with these measures (Tarampi, Heydari, \& Hegarty, 2016), males produced lower error scores than females on the SOT, $t(66)=$ 2.02, $p=.05$, and outperformed females on the MRM, $t(66)=$ $3.09, p=.003$. There was a nonsignificant trend such that males reported better sense of direction than females, $t(66)=$ $1.80, p=.08$ (cf. Hegarty et al., 2006). Consistent with previous research (Lawton, 1994, 1996), females were more likely than males to indicate use of route-based strategy on the WSS, $t(66)=3.75, p<.001$, and females reported more spatial anxiety than males, $t(66)=2.33, p=.02$. However, no differences were found between males and females for self-reported use of the orientation strategy, $t(66)=1.40, p=.17$. Finally, males $(M=3.14, S D=1.79)$ reported playing video games more frequently than females $(M=1.83, S D=1.01), t(66)=$ $5.15, p<.001$.

Navigation performance Successful navigation to the goal location occurred on $98.6 \%$ of the 12 shortcut-available trials. A strict coding of the main strategies as shortcut or learned route accounted for over half of the trials (mean of 6.99 out of 12). Including liberal coding, an average of 4.81 of the 12 paths were coded as shortcuts, 2.96 were coded as learned routes, and 2.25 were coded as reversed learned routes. In addition, 1.44 of the 12 trials were coded as wandering and an average of 0.54 of the 12 trials, that is, $4.5 \%$ of trials were uncodable.

As seen in Fig. 4, based on the SI measure, participants showed a wide range of strategy preference, ranging from always taking learned routes to always taking shortcuts $(M=$ $.61, S D=.30$; range $=.08-1.00)$ as in previous research on this task (Marchette et al., 2011).

\section{Sex differences in solution index and navigation efficiency As} predicted, males $(M=.72, S D=.29)$ showed a significantly greater preference for shortcuts than females $(M=.54, S D=$ $.25), t(66)=2.70, p=.009, d=.66 .^{3}$ As predicted, sex differences were also found in efficiency such that males $(Z$-score $M$ $=-0.27, S D=.43)$ took less time to reach the goal than females $(Z$-score $M=.19, S D=.51), t(66)=3.93, p<.001$, $d=.98$. Efficiency correlated with SI such that those who took more shortcuts reached the goal faster, $r(66)=-.56, p<.001$. To assess whether the difference in efficiency was due to differences in strategy as measured by the SI, a univariate ANCOVA was carried out comparing the efficiency of males

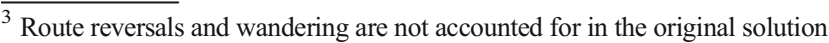
index measure. A second analysis was carried out incorporating all successful trials. Results did not differ appreciably between the solution index measures. Therefore, the original SI measure is used here for consistency with previous research.
}

and females while controlling for navigation preference using SI. There was a significant effect of sex on efficiency such that males were more efficient than females, even after controlling for navigation preference (SI), $F(1,65)=7.92, p=.006, \eta_{\mathrm{p}}{ }^{2}=$ .11. This indicates that sex difference in efficiency was not only due to solution strategy as measured by the solution index.

Sex differences in route selection Figure 5 plots the average number of shortcuts, learned routes, reversed learned routes, and wandering trials taken by males and females. To test for differences between males and females for each strategy, Mann-Whitney $U$ tests were conducted, with alpha adjusted for multiple comparisons using Bonferonni correction $(p=$ .0125 ). As predicted, males traversed significantly more shortcuts than females, $U=355.50, p=.01, d=.67$, and females traversed significantly more learned routes than males, $U=$ $361.00, p=.01, d=.54$. No sex differences were found for reversing the learned route, $U=495.50, p=.41$. Interestingly, there was a trend for females to wander more than males, $U=$ $355.50, p=.06, d=.57$. Specifically, 28 females wandered on at least one trial (average of 2.5 trials), while 17 males wandered on at least one trial (average of 1.65 trials).

Correlations of strategy and efficiency with psychometric measures Correlations of strategy and efficiency with the psychometric measures are shown in Table 2. Of the two measures of perspective taking, only the MRM test correlated with SI, such that participants with better perspective-taking ability took more shortcuts. Participants who reported route-based strategies on the WSS also took more learned routes in the DSP, providing limited evidence that self-report measures are consistent with objective measures of navigation strategy. However, SI does not reliably correlate with other self-report measures of strategy or with SBSOD. In contrast, ability measures correlated significantly with measures of efficiency. Specifically, participants with a better self-reported sense of direction and better perspective-taking ability (as measured by both the MRM and SOT tests) took less time to reach the goal locations, and participants who reported using route strategies in everyday life were less efficient.

\section{Discussion}

Experiment 1 provides objective evidence for a reliable sex difference in strategy in the DSP whereby males took significantly more shortcuts, and females were more likely to take learned routes. Further, Experiment 1 indicated large sex differences in navigation efficiency (measured by time to travel to goal locations and directness of travel). The sex difference in efficiency was somewhat related to differences in strategy as measured by the solution index (SI); however, SI did not account for all of the variance in efficiency. Moreover, 
Table 1 Descriptive statistics for the paper-and-pencil measures in each experiment

\begin{tabular}{|c|c|c|c|c|c|c|c|c|}
\hline \multirow[b]{3}{*}{ Measure } & \multicolumn{4}{|c|}{ Experiment 1} & \multicolumn{4}{|c|}{ Experiment 2} \\
\hline & \multicolumn{2}{|l|}{ Males } & \multicolumn{2}{|c|}{ Females } & \multicolumn{2}{|l|}{ Males } & \multicolumn{2}{|c|}{ Females } \\
\hline & $M$ & $S D$ & $M$ & $S D$ & $M$ & $S D$ & $M$ & $S D$ \\
\hline SOT & 27.03 & 21.38 & 36.59 & 17.61 & 27.23 & 23.36 & 42.39 & 27.35 \\
\hline Money & 12.29 & 5.34 & 8.83 & 3.91 & 12.86 & 4.78 & 8.61 & 4.57 \\
\hline SBSOD & 4.30 & 1.06 & 3.87 & 0.90 & 4.09 & 1.11 & 3.80 & 1.02 \\
\hline WSS Orientation & 2.83 & 0.69 & 2.62 & 0.53 & 2.88 & 0.80 & 2.37 & 0.62 \\
\hline WSS Route & 3.08 & 0.65 & 3.67 & 0.62 & 3.22 & 0.82 & 3.31 & 0.76 \\
\hline WSS Anxiety & 2.68 & 0.84 & 3.12 & 0.70 & 2.46 & 0.67 & 2.75 & 0.73 \\
\hline
\end{tabular}

Note. SOT = Spatial Orientation Test; Money = Money Road Map Test; SBSOD = Santa Barbara Sense of Direction Scale; WSS = Wayfinding Strategy Scale. SOT is an error score such that lower scores indicate better performance

although efficiency in finding the goal location had reliable correlations with psychometric measures of strategy and ability, the solution index measure, which takes account of shortcuts and learned routes, provided only limited evidence that
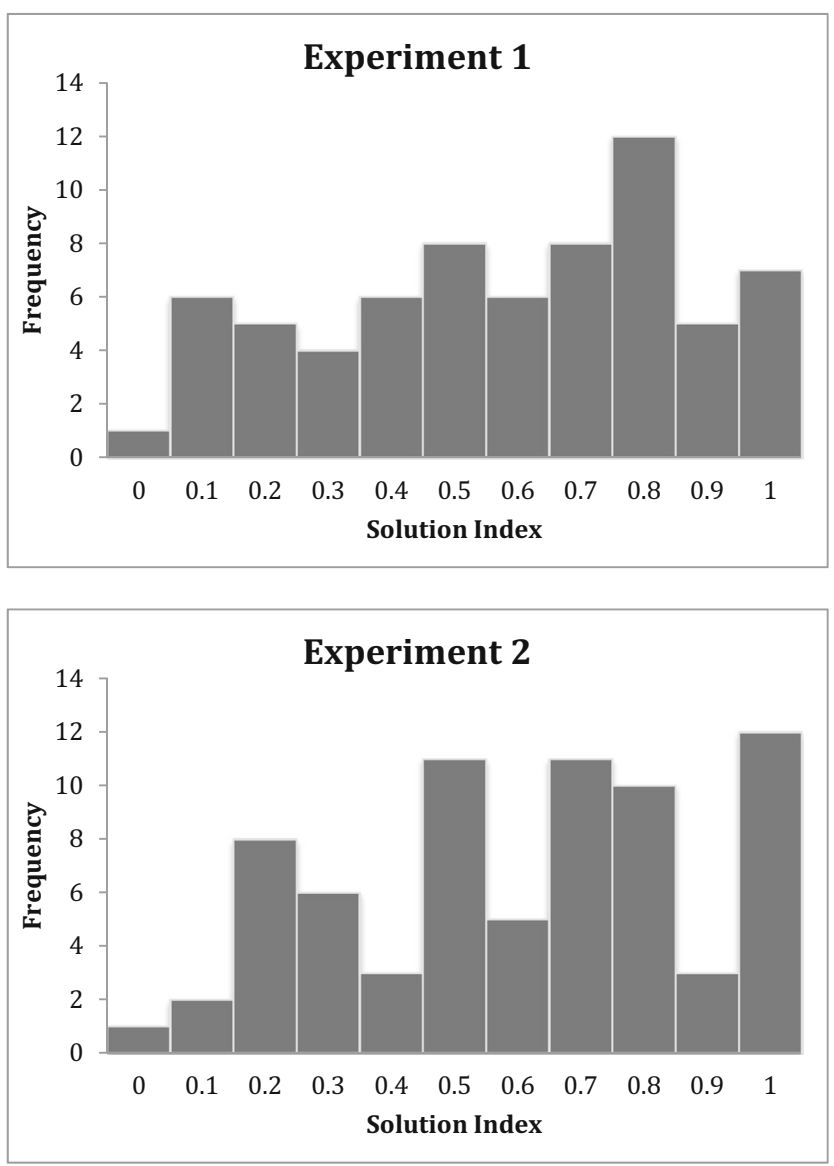

Fig. 4 Histogram of solution index of Experiments 1 and 2. Note. Values closer to zero indicate use of learned routes, whereas values closer to one indicate use of shortcut routes self-reports of navigation strategy are related to objective measures of strategy.

\section{Experiment 2}

Experiment 1 indicated large sex differences in navigation efficiency. Although the sex difference in solution index was smaller, it was in the medium range (Cohen, 1992). In Experiment 2 we attempted to replicate and extend these findings while addressing some limitations of Experiment 1.

First, the environment in Experiment 1 was unlike the real world in that it lacked distal landmarks such as trees or mountains. This contrasts with at least two previous studies of using the DSP in which distal cues (mountains) were present in the environment (Furman et al., 2014; Weisberg \& Newcombe, 2016). Other research suggests that distal landmarks can be used to navigate and that there are individual and sex differences in their use (Chai \& Jacobs, 2009; Iaria et al., 2003; Steck \& Mallot, 2000). For instance, some people are more affected when distal cues are removed, suggesting individual differences in use of these cues (Iaria et al., 2003). Moreover, although women in general are more likely to use landmarks in navigation (Coluccia \& Louse, 2004), men report more dependence on distal landmarks than do women (Lawton, 1994), and Chai and Jacobs (2009) showed that males were more aided by directional (distal) cues in a virtual version of the Morris water maze, suggesting that males may pay more attention to these cues than do females.

Experiment 2 compared performance in the DSP in environments with and without distal landmarks (features that were exterior to the environment in which people navigated). We investigated whether the presence of distal landmarks affected navigation strategy and efficiency. Previous research suggests several possible outcomes. First, when given stable distal landmarks, participants may be more able to construct 


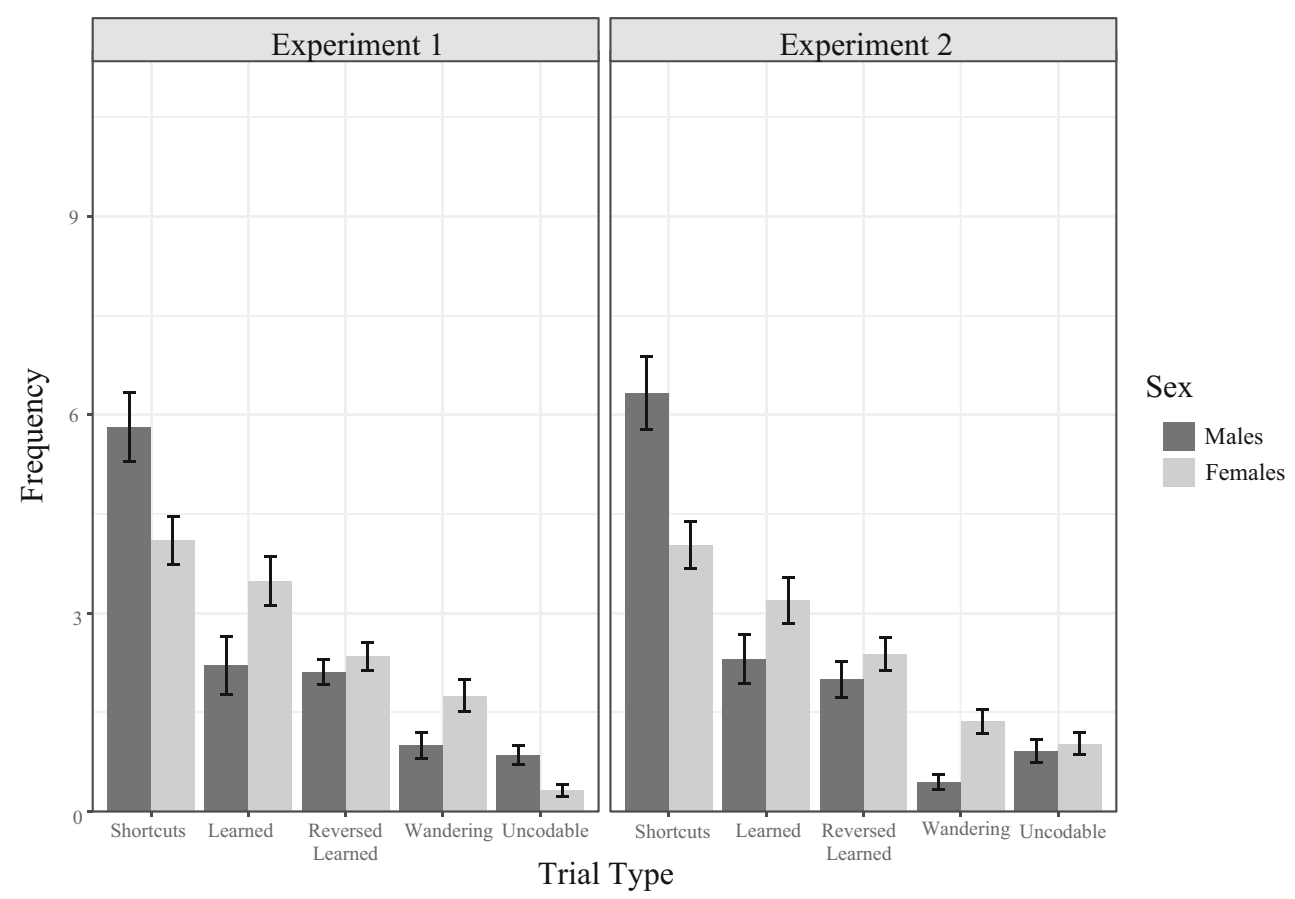

Fig. 5 Route selection coding by sex in each experiment. Error bars show the standard error of the mean

survey representations of the environment so that they take more shortcuts in general navigating between targets more efficiently. Second, in a situation where local and distal cues exist, people may ignore the distal cues altogether relying instead on the local cues. A third possibility is that there are individual differences in use of distal cues. Previous research suggests that females would be more likely to ignore distal cues (Chai \& Jacobs, 2009; Lawton, 1994). Therefore, adding stable landmarks exterior to the environment may increase sex differences, by increasing both efficiency and the use of shortcuts by men.

This study also addressed two other limitations of Experiment 1: Some of the local maze landmarks in Experiment 1 (e.g., floating soccer ball) were not ecologically

Table 2 Correlation of dependent measures with individual difference measures in each experiment

\begin{tabular}{lccccc}
\hline & \multicolumn{2}{l}{ Experiment 1} & & \multicolumn{2}{l}{ Experiment 2} \\
\cline { 2 - 3 } \cline { 5 - 6 } \cline { 5 - 6 } & $\begin{array}{l}\text { Strategy } \\
\text { (SI) }\end{array}$ & $\begin{array}{l}\text { Efficiency } \\
\text { (Time) }\end{array}$ & & $\begin{array}{l}\text { Strategy } \\
\text { (SI) }\end{array}$ & $\begin{array}{l}\text { Efficiency } \\
\text { (Time) }\end{array}$ \\
\hline Money Road Map test & $0.30^{* *}$ & $-0.29^{*}$ & & 0.12 & $-0.32^{* *}$ \\
Spatial Orientation Test & 0.03 & $0.38^{* * *}$ & & 0.02 & 0.16 \\
SBSOD & 0.15 & $-0.30^{* *}$ & & 0.08 & $-0.26^{*}$ \\
WSS Orientation & -0.03 & -0.05 & & 0.01 & $-0.24^{*}$ \\
WSS Route & $-0.26^{*}$ & $0.26^{*}$ & & -0.14 & -0.03 \\
WSS Spatial Anxiety & -0.16 & 0.22 & -0.09 & 0.15 \\
\hline
\end{tabular}

Note. $\mathrm{SI}=$ solution index. ${ }^{*} p<.05, * * p \leq .01, * * * p=.001$ valid, and participants were not required to learn the names of the landmarks in advance of the trials. In Experiment 2 we replaced some of the landmarks with more ecologically valid landmarks and had participants learn the names of the landmarks prior to the DSP trials.

\section{Method}

\section{Participants}

Participants were 82 University of California, Santa Barbara, undergraduates (43 female) who participated in return for course credit. Ten participants were excluded from analysis due to motion sickness $(N=7)$, experimenter error $(N=2)$, and lack of English fluency $(N=1)$. The analyses were based on data from 72 participants, 18 males and 18 females, who participated in each condition (distal landmarks vs. no distal landmarks).

\section{Design}

The experiment had a 2 (condition: distal landmarks, no distal landmarks) $\times 2$ (sex: male, female) between-subjects experimental design.

\section{Materials}

The computer hardware and software were unchanged from Experiment 1. Two changes were made to the maze environment. First, unique distal cues were placed at each cardinal 
direction outside of the maze environment (a fir tree on one side, a palm tree on a second side, an oak tree on a third side, and two fir trees on the fourth side; see Fig. 2b). These trees were visible during encoding and testing trials. Secondly, three of the 11 local maze landmarks were replaced with more ecologically valid landmarks (a trash can replaced the car; a mailbox replaced the duck; and a picnic table replaced the soccer ball).

\section{Procedure}

Two changes were made to the procedure of Experiment 1. First, participants spoke the name of each object aloud on the first learning tour in order to ensure familiarity with the names by which we would refer to these landmarks in the experimental trials. Second, breaks were given before starting the testing trials and after half of the trials in order to reduce the chance of motion sickness. Finally the SBSOD, WSS, and a question about video-game experience were administered online rather than on paper.

\section{Coding}

Using the same mathematically formalized criteria for liberal coding as in Experiment 1, trials were coded as shortcuts, learned routes, reversed learned route, wandering, and uncodable. The mean length of liberal shortcut trials was $65 \%$ of the learned route, and these trials overlapped $40 \%$ with the learned route. Trials classified as liberal learned route had $80 \%$ overlap with the learned route, and trials classified as reversed learned liberal overlapped on average $89 \%$ with the reverse of the learned route.

Solution index and efficiency measures were computed as in Experiment 1. Again, there was a strong correlation between the distance and time efficiency measures, $r(80)=$ $.95, p<.001$, and thus the measure of efficiency that we analyzed was average time to reach the goal location.

\section{Results}

Participants in the two conditions (with vs. without distal landmarks) did not differ on perspective-taking ability, SBSOD, WSS route or anxiety, or video game use, all $t \mathrm{~s}(70) \leq 1.73, p \geq$ .09; however, participants in the control (no distal landmarks) condition were more likely to report an orientation strategy on the WSS, $t(70)=2.10, p=.04$.

Sex differences in ability and self-report measures As can be seen in Table 1, males had lower error scores on the SOT than did females, $t(70)=2.58, p=.01$, and correctly identified more turns in the MRM, $t(70)=3.85, p<.001$. Males indicated using more orientation strategies than did females in the WSS, $t(70)=$ $3.05, p=.003$. No differences were found between males and females in SBSOD, WSS route strategy usage, or spatial anxiety, $t(70) \leq 1.74, p \geq .09$. Finally, males $(M=3.17, S D=.94)$ reported playing video games more frequently than did females $(M=2.11, S D=.90), t(66)=4.81, p<.001$.

Navigation performance Successful navigation occurred on $11.67(S D=.58)$ out of 12 trials. A strict coding of the main strategies as shortcut or learned route accounted for over half of the trials (mean of 7.27 out of 12). Including liberal coding, an average of 5.18 of the 12 paths were coded as shortcuts, 2.75 were coded as learned path, and 2.19 were coded as reversed learned path. In addition, 0.90 of the 12 trials were coded as wandering. Finally, trials that did not fall into any of these categories were classified as uncodable (an average of 0.97 of the 12 trials; that is, $7.99 \%$ of all trials).

As shown in Fig. 4, participants' SI scores showed a range of strategies $(M=.64, S D=.27$; range $=.09-1.00)$, replicating Experiment 1 and previous research.

Effects of landmark condition and sex on solution index Descriptive statistics broken down by landmark condition and sex can be found in Table 3. As indicated by a univariate 2 (distal cue, no cue) $\times 2$ (sex) ANOVA, there was a sex difference in solution index. As in Experiment 1, males $(M$ $=.71, S D=.27$ ) showed more shortcut preference in SI than did females $(M=.56, S D=.24), F(1,68)=5.78, p=.02, \eta_{\mathrm{p}}{ }^{2}=$ $.08, d=.67$. However, the solution index did not differ significantly between the distal landmarks group $(M=.63, S D=$ $.27)$ and the control group $(M=.65, S D=.27), F(1,68)=.08$, $p=.78$. There was no significant interaction between sex and condition, $F(1,68)=.28, p=.60$.

Effects of landmark condition and sex on efficiency measures A univariate ANOVA indicated that males $(M=-.30, S D=$ .35) were significantly more efficient in finding the goal

Table 3 Descriptive statistics of all objective navigation measures in Experiment 2

\begin{tabular}{|c|c|c|c|c|}
\hline \multirow[b]{3}{*}{ Measure } & \multicolumn{4}{|l|}{ Experiment 2} \\
\hline & \multicolumn{2}{|l|}{ Distal cues } & \multicolumn{2}{|l|}{ No cues } \\
\hline & Males & Females & Males & Females \\
\hline Shortcuts & $5.61(3.13)$ & $3.61(1.58)$ & 7.05 (3.39) & 4.44 (2.57) \\
\hline Learned & $2.33(2.28)$ & $2.78(1.66)$ & $2.28(2.30)$ & $3.61(2.45)$ \\
\hline Reversal & $2.44(1.38)$ & $2.78(1.56)$ & $1.56(1.79)$ & $2.00(1.37)$ \\
\hline Wandering & $0.50(0.71)$ & $1.44(1.15)$ & $0.38(0.61)$ & $1.28(1.02)$ \\
\hline Uncodable & $1.11(0.90)$ & $1.39(0.98)$ & $0.72(1.13)$ & $0.67(0.84)$ \\
\hline Solution index & $0.69(0.29)$ & $0.57(0.24)$ & $0.71(0.27)$ & $0.56(0.24)$ \\
\hline Efficiency & $-0.24(0.33)$ & $0.43(0.48)$ & $-0.36(0.37)$ & $0.17(0.46)$ \\
\hline
\end{tabular}

Note. The efficiency measure is based on time and is a $Z$-score average. Standard deviation is presented in parentheses 
locations than were females $(M=.30, S D=.49), F(1,68)=$ $37.47, p<.001, \eta_{\mathrm{p}}{ }^{2}=.36$. The control group $(M=-.10, S D=$ $.53)$ was also more efficient than the distal cue group $(M=.10$ $S D=.49), F(1,68)=4.12, p=.05, \eta_{\mathrm{p}}{ }^{2}=.06$, indicating that, if anything, the additional distal landmarks impeded navigation. The interaction was not significant, $F(1,68)=.52, p=47$.

As in Experiment 1, efficiency in Experiment 2 was significantly correlated with SI such that those who took more shortcuts travelled less distance, $r(68)=-.51, p<.001$. A univariate ANCOVA was carried out comparing efficiency of males and females while controlling for navigation preference using SI. There was a significant effect of sex on efficiency such that males were more efficient than females, even after controlling for solution index, $F(1,67)=26.28, p<.001, \eta_{\mathrm{p}}{ }^{2}=.28$. Again, this indicates that the sex difference in efficiency was not only due to solution strategy as measured by the SI.

\section{Effects of landmark condition and sex on route selection strat-} egies Mann-Whitney $U$ tests were used to compare performance of each route selection category (shortcut, learned route, reverse learned route, wandering) by condition and by sex, adjusting alpha using Bonferroni corrections $(p=.0125)$ for multiple comparisons. Figure 5 provides the descriptive statistics for each strategy. Males traversed significantly more shortcuts than females, $U=384.00, p=.003, d=.83$. No sex differences were found in traversing learned routes, $U=471.00, p=.04, d=$ .40 , nor reversing the learned route, $U=560.00, p=.31$. Interestingly, females were significantly more likely to wander than males, $U=333.50, p<.001, d=1.04$. No differences between conditions were found for shortcuts, learned routes, nor wandering, all $U_{\mathrm{s}} \geq 529.50$, all $p \mathrm{~s} \geq .18$. However, there was a nonsignificant trend for the distal group was to reverse learned routes more than the control group, $U=447.50, p=.02$.

Correlations of strategy and efficiency with psychometric measures As shown in Table 2, scores on the MRM correlated with efficiency such that participants with better perspective taking ability as measured by this task reached the goal faster. However, in general, the correlations of the individual differences measures with performance on the DSP were lower than in Experiment 1.

\section{Discussion}

Experiment 2 replicated the main results of Experiment 1, namely a large sex difference in navigation efficiency and a medium sex difference in navigation strategy. Males took significantly more shortcuts, and females wandered more; however, in contrast to Experiment 1, females did not take significantly more learned routes. While Experiment 2 generalized the results of Experiment 1 to an environment with extra maze cues, in general, few participants in the distal landmark condition mentioned noticing or using these landmarks, and indeed they had little effect on navigation strategy and efficiency. Further, the improvements to the design of this experiment (more ecologically valid landmarks and naming the landmarks aloud) had little effect.

The correlations of objective measures of strategy and efficiency with psychometric measures were smaller than Experiment 1, especially for strategy. However, a similar story emerged concerning the nature of strategy and efficiency such that efficiency was more significantly correlated with these psychometric measures.

\section{General discussion}

Across two experiments, we used the dual-solution paradigm (DSP) to examine the strategies that people use to navigate to a goal location in a learned environment, sex differences in navigation strategy, how these differences affect navigation efficiency, and how they relate to other measures of largescale spatial ability.

\section{Navigation in the dual-solution paradigm}

Our results corroborate recent findings indicating that in the DSP participants mainly utilized novel, unlearned shortcuts and learned routes (Furman et al., 2014; Marchette et al., 2011). Although previous research using the DSP did not report other possible solutions, we found that on some trials participants navigated by routes that varied in efficiency but were neither the shortest route nor the learned route. Specifically, occasionally participants travelled by backtracking the originally learned route and shortcutting across that route. We also found that participants sometimes reached the goal location by wandering. This suggests that the range of behaviors in the DSP might be richer than is reflected by the solution index dependent measure, which only considers learned routes and shortcuts.

\section{Sex differences}

The research presented here indicates a medium-sized sex difference in the solution index (SI) that was almost equal in size (.66 vs .67) in the two experiments. In line with self-report findings (Lawton, 1994, 1996; Lawton \& Kallai, 2002), males were more likely to take shortcuts and females were more likely to take learned routes. This adds to the literature on sex differences in navigation strategy (Astur et al., 2004; Lawton, 1994; Sandstrom et al., 1998) and provides important new evidence of sex differences in navigation strategy in a laboratory study based on objective measures as opposed to self-report measures or measures of ability.

Consistent with previous research, a large sex difference was found in navigation efficiency, as measured by time to navigate to a goal location and directness of the route taken 
(Astur et al., 2004; Moffat et al., 1998; Sandstrom et al., 1998). A new question addressed by the present research is the extent to which greater efficiency of navigation is due to differences in route selection as measured by the solution index of the DSP. In both experiments, males were significantly more efficient than females, even after controlling for the effects of strategy. This may be partly because wandering and reversing the learned route are not accounted for within the solution index measure. Given that wandering reflects inadequate knowledge of landmark locations within the environment, it suggests that another source of the sex difference in environmental spatial cognition, beyond a navigator's preferences, may be related to the difference in the ability to learn the layout of an environment with a given amount of experience. It is also possible that the sex difference in efficiency is due in part to facility with the interface or navigation in virtual environments, as men tend to spend more time playing video games (Hegarty et al., 2006; Waller, 2000).

\section{Distal landmarks}

The experimental manipulation of adding distal landmarks exterior to the environment in Experiment 2 had little effect on navigation strategy or efficiency. Although previous research has shown that distal landmarks (e.g., mountains) can be used to navigate in a virtual environment (Iaria et al., 2003; Steck \& Mallot, 2000), there was no evidence in our experiment that participants used the distal cues for more efficient navigation. Although our distal landmarks were relatively distinct species of trees, they occupied only a small part of the visual field, so it is possible that participants did not notice them. In fact, in a posttask questionnaire, only four participants specifically mentioned using the trees to navigate. In contrast, Steck and Mallot (2000) familiarized participants with their distal landmarks to ensure participants noticed them. The one significant effect of the distal cues was that reversing the learned route became somewhat more common when these cues were added, and removing the participants who mentioned using the cues from the analyses reduced this effect. It remains possible that the tree landmarks in Experiment 2 acted as local cues to travel near (e.g., another route object) rather than distal cues, as intended. In order to further examine the effects of landmarks beyond the boundaries of the environment, future studies should examine the effects of larger scale, more differentiated, and more distal landmarks such as mountains.

\section{Objective versus subjective measures}

In this research, we found partial support for a relationship between self-reported navigation strategy and the strategy that people used to navigate in a virtual environment. In Experiment 1, self-reported route strategy was significantly correlated with taking more learned routes as well as longer travel distance. However, this pattern of results did not extend to Experiment 2, and these correlations were not large. These results raise questions about the validity of the Wayfinding Strategy Scale as a measure of navigation strategy. This scale asks participants about a specific past navigation event. This event may be salient precisely because it was atypical. Thus, responses to this questionnaire may differ from one's everyday strategies in navigation. Further, this questionnaire refers to everyday navigation methods that may be outdated, such as the use of paper maps and other forms of communication that have changed since the advent of global positioning systems and smartphones. For instance, many of the route subscale questions are contingent upon the participant asking for directions (e.g., "Before starting, I asked for directions telling me how many streets to pass before making each turn"), whereas these days there is a stronger possibility of participants using mobile navigation devices (e.g., smartphones). It is possible that participants do not know what they would do in this situation. Modification of this questionnaire may increase its usefulness in future research.

The present studies were conducted in desktop virtual environments. Although virtual environments have the advantage of experimental control, and results are often consistent in virtual and real environments (Richardson, Montello, \& Hegarty, 1999; Weisberg et al., 2014) there are differences in everyday navigation and navigation in desktop virtual environments. One important difference is that navigation in the real world includes self-motion perception, whereas navigation in desktop VR does not (cf. Hegarty et al., 2006). Previous research has shown navigation tasks are aided by kinesthetic information that arises from physical movement such as rotations (Allen, Kirasic, Rashotte, \& Haun, 2004; Chance, Gaunet, Beall, \& Loomis, 1998; Kearns, Warren, Duchon, \& Tarr, 2002;). While virtual environments have the advantage of experimental control, it is also important to examine strategy differences in navigation in real environments, in which there is more cost in terms of physical effort of taking a less efficient route and in which path integration is another available navigation strategy.

\section{Strategy versus ability}

An important question raised by this research is whether navigation strategy is determined by one's ability to construct a survey-like representation of the environment (i.e., a cognitive map) or whether other factors affect strategy. A person with metrically accurate survey knowledge of the environment should be able to find and take shortcuts, but a shortcutting strategy is not available to a person with only route knowledge. However, strategy selection may depend on preferences, as well as the environmental knowledge someone possesses. For instance, route choice might depend on individual 
characteristics such as willingness to take risks or situational factors such as time pressure. While shortcutting indicates survey knowledge, taking learned routes may be due to preference, a lack of environmental knowledge, or some combination of these factors. Here, we found that the objective strategy measure, the solution index, correlated only weakly with measures of navigation ability (SBSOD), and perspectivetaking ability (SOT and MRM), which are related to ability to construct a survey representation of an environment. While these findings suggest that the strategy an individual uses to navigate is not entirely dependent on their large-scale ability it will be important to examine this more directly in future research by examining the relationship between navigation strategy and more direct measures of ability to construct a survey representation from navigation experience.

\section{Conclusion}

We investigated sex differences in the dual-solution paradigm (DSP), which can be used to objectively measure both navigation strategy and efficiency. A large spread in strategy usage was found across participants. Shortcuts and learned routes were the primary strategies used in the DSP, but other behaviors were observed such as route reversals and wandering. Importantly, sex differences were found in both route selection and navigation efficiency. Men were more likely to take shortcuts in the DSP, as expected on the basis of self-report measures. Correlations between self-report and objective measures of strategies were weak, raising questions about the validity or comparability of existing self-report and objective measures. Overall, this research indicates that the sex difference in navigation efficiency is large, and is partly related to navigation strategy.

Author notes This research was supported by a grant to M.H. from the Academic Senate of the University of California, Santa Barbara. All data are available upon request and navigational data files are available online at www.osf.io/wce8d. The authors would like to thank David Sañosa for assistance in creation of the virtual environment as well as Dan Montello, Elizabeth R. Chrastil, Peri Gunalp, and four anonymous reviewers for providing critical comments on an earlier version of this manuscript.

\section{References}

Allen, G. L., Kirasic, K. C., Rashotte, M. A., \& Haun, D. B. (2004). Aging and path integration skill: Kinesthetic and vestibular contributions to wayfinding. Perception \& Psychophysics, 66(1), 170179.
Astur, R. S., Ortiz, M. L., \& Sutherland, R. J. (1998). A characterization of performance by men and women in a virtual Morris water task: A large and reliable sex difference. Behavioral Brain Research, 93(1), 185-190.

Astur, R. S., Tropp, J., Sava, S., Constable, R. T., \& Markus, E. J. (2004). Sex differences and correlations in a virtual Morris water task, a virtual radial arm maze, and mental rotation. Behavioural Brain Research, 15l(1), 103-115.

Chai, X. J., \& Jacobs, L. F. (2009). Sex differences in directional cue use in a virtual landscape. Behavioral Neuroscience, 123(2), 276.

Chance, S. S., Gaunet, F., Beall, A. C., \& Loomis, J. M. (1998). Locomotion mode affects the updating of objects encountered during travel: The contribution of vestibular and proprioceptive inputs to path integration. Presence, 7(2), 168-178.

Chrastil, E. R., \& Warren, W. H. (2015). Active and passive spatial learning in human navigation: Acquisition of graph knowledge. Journal of Experimental Psychology: Learning, Memory, and Cognition, 41(4), 1162-1178.

Cohen, J. (1992). A power primer. Psychological Bulletin, 112(1), 155159.

Coluccia, E., \& Louse, G. (2004). Gender differences in spatial orientation: A review. Journal of Environmental Psychology, 24(3), 329340.

Cutmore, T. R., Hine, T. J., Maberly, K. J., Langford, N. M., \& Hawgood, G. (2000). Cognitive and gender factors influencing navigation in a virtual environment. International Journal of Human-Computer Studies, 53(2), 223-249.

Foo, P., Warren, W. H., Duchon, A., \& Tarr, M. J. (2005). Do humans integrate routes into a cognitive map? Map-versus landmark-based navigation of novel shortcuts. Journal of Experimental Psychology: Learning, Memory, and Cognition, 31(2), 195-215.

Furman, A. J., Clements-Stephens, A. M., Marchette, S. A., \& Shelton, A. L. (2014). Persistent and stable biases in spatial learning mechanisms predict navigational style. Cognitive, Affective, \& Behavioral Neuroscience, 14(4), 1375-1391.

Grön, G., Wunderlich, A. P., Spitzer, M., Tomczak, R., \& Riepe, M. W. (2000). Brain activation during human navigation: gender-different neural networks as substrate of performance. Nature Neuroscience, 3(4), 404-408.

Hegarty, M., Montello, D. R., Richardson, A. E., Ishikawa, T., \& Lovelace, K. (2006). Spatial abilities at different scales: Individual differences in aptitude-test performance and spatial-layout learning. Intelligence, 34(2), 151-176.

Hegarty, M., Richardson, A. E., Montello, D. R., Lovelace, K., \& Subbiah, I. (2002). Development of a self-report measure of environmental spatial ability. Intelligence, 30(5), 425-447.

Hegarty, M., \& Waller, D. (2004). A dissociation between mental rotation and perspective-taking spatial abilities. Intelligence, 32(2), 175191.

Iaria, G., Petrides, M., Dagher, A., Pike, B., \& Bohbot, V. D. (2003). Cognitive strategies dependent on the hippocampus and caudate nucleus in human navigation: variability and change with practice. The Journal of Neuroscience, 23(13), 5945-5952.

Ishikawa, T., \& Montello, D. R. (2006). Spatial knowledge acquisition from direct experience in the environment: Individual differences in the development of metric knowledge and the integration of separately learned places. Cognitive Psychology, 52(2), 93-129.

Kearns, M. J., Warren, W. H., Duchon, A. P., \& Tarr, M. J. (2002). Path integration from optic flow and body senses in a homing task. Perception, 31(3), 349-374.

Labate, E., Pazzaglia, F., \& Hegarty, M. (2014). What working memory subcomponents are needed in the acquisition of survey knowledge? Evidence from direction estimation and shortcut tasks. Journal of Environmental Psychology, 37, 73-79. 
Lambrey, S., \& Berthoz, A. (2007). Gender differences in the use of external landmarks versus spatial representations updated by selfmotion. Journal of Integrative Neuroscience, 6(03), 379-401.

Lawton, C. A. (1994). Gender differences in way-finding strategies: Relationship to spatial ability and spatial anxiety. Sex Roles, 30(11/ 12), 765-779.

Lawton, C. A. (1996). Strategies for indoor wayfinding: The role of orientation. Journal of Environmental Psychology, 16(2), 137-145.

Lawton, C. A., Charleston, S. I., \& Zieles, A. S. (1996). Individual-and gender-related differences in indoor wayfinding. Environment and Behavior, 28(2), 204-219.

Lawton, C. A., \& Kallai, J. (2002). Gender differences in wayfinding strategies and anxiety about wayfinding: A cross-cultural comparison. Sex Roles, 47(9/10), 389-401.

Maguire, E. A., Woollett, K., \& Spiers, H. J. (2006). London taxi drivers and bus drivers: A structural MRI and neuropsychological analysis. Hippocampus, 16(12), 1091-1101.

Marchette, S. A., Bakker, A., \& Shelton, A. L. (2011). Cognitive mappers to creatures of habit: differential engagement of place and response learning mechanisms predicts human navigational behavior. The Journal of Neuroscience, 31(43), 15264-15268.

Moffat, S. D., Hampson, E., \& Hatzipantelis, M. (1998). Navigation in a "virtual" maze: Sex differences and correlation with psychometric measures of spatial ability in humans. Evolution and Human Behavior, 19(2), 73-87.

Money, J., Alexander, D., \& Walker, H. T. (1965). A standardized roadmap test of direction sense: Manual. Baltimore, MD: Johns Hopkins Press.

Montello, D. R., Lovelace, K. L., Golledge, R. G., \& Self, C. M. (1999). Sex-related differences and similarities in geographic and environmental spatial abilities. Annals of the Association of American Geographers, 89(3), 515-534.

O'Keefe, J., \& Nadel, L. (1978). The hippocampus as a cognitive map. Oxford, UK: Clarendon Press.

Packard, M. G., \& McGaugh, J. L. (1996). Inactivation of hippocampus or caudate nucleus with lidocaine differentially affects expression of place and response learning. Neurobiology of Learning and Memory, 65(1), 65-72.

Pazzaglia, F., Cornoldi, C., \& De Beni, R. (2000). Differenze individuali nella rappresentazione dello spazio e nell'abilita di orientamento: Presentazione di un questionario autovalutativo [Individual differences in the representation of space and ability to orientate: Presentation of a self-assessment questionnaire]. Giornale Italiano Di Psicologia, 27(3), 627-650.
Richardson, A. E., Montello, D. R., \& Hegarty, M. (1999). Spatial knowledge acquisition from maps and from navigation in real and virtual environments. Memory \& Cognition, 27(4), 741-750.

Sandstrom, N. J., Kaufman, J., \& Huettel, S. A. (1998). Males and females use different distal cues in a virtual environment navigation task. Cognitive Brain Research, 6(4), 351-360.

Saucier, D. M., Green, S. M., Leason, J., MacFadden, A., Bell, S., \& Elias, L. J. (2002). Are sex differences in navigation caused by sexually dimorphic strategies or by differences in the ability to use the strategies?. Behavioral Neuroscience, 116(3), 403-410.

Schinazi, V. R., Nardi, D., Newcombe, N. S., Shipley, T. F., \& Epstein, R. A. (2013). Hippocampal size predicts rapid learning of a cognitive map in humans. Hippocampus, 23(6), 515-528.

Sholl, M. J., Acacio, J. C., Makar, R. O., \& Leon, C. (2000). The relation of sex and sense of direction to spatial orientation in an unfamiliar environment. Journal of Environmental Psychology, 20(1), 17-28.

Siegel, A. W., \& White, S. H. (1975). The development of spatial representations of large-scale environments. Advances in Child Development and Behavior, 10, 9-55.

Silverman, I., Choi, J., Mackewn, A., Fisher, M., Moro, J., \& Olshansky, E. (2000). Evolved mechanisms underlying wayfinding: Further studies on the hunter-gatherer theory of spatial sex differences. Evolution and Human Behavior, 21(3), 201-213.

Steck, S. D., \& Mallot, H. A. (2000). The role of global and local landmarks in virtual environment navigation. Presence: Teleoperators and Virtual Environments, 9(1), 69-83.

Tarampi, M. R., Heydari, N., \& Hegarty, M. (2016). A tale of two types of perspective taking: Sex differences in spatial ability. Psychological Science, 27(11), 1507-1516.

Waller, D. (2000). Individual differences in spatial learning from computer-simulated environments. Journal of Experimental Psychology: Applied, 6(4), 307-321.

Weisberg, S. M., \& Newcombe, N. S. (2016). How do (some) people make a cognitive map? Routes, places, and working memory. Journal of Experimental Psychology: Learning, Memory, and Cognition, 42(5), 768-785.

Weisberg, S. M., Schinazi, V. R., Newcombe, N. S., Shipley, T. F., \& Epstein, R. A. (2014). Variations in cognitive maps: Understanding individual differences in navigation. Journal of Experimental Psychology: Learning, Memory, and Cognition, 40(3), 669-682.

Woollett, K., \& Maguire, E. A. (2011). Acquiring "the Knowledge" of London's layout drives structural brain changes. Current Biology, 21(24), 2109-2114. 